\title{
Determination of the rotary fatigue life of NiTi alloy wires
}

\author{
André Carvalho $^{\mathrm{a}}$, Diogo Montalvão ${ }^{\mathrm{b}}$, Manuel Freitas ${ }^{\mathrm{a}, *}$, Luis Reis ${ }^{\mathrm{a}}$, Manuel Fonte ${ }^{\mathrm{a}, \mathrm{c}}$ \\ a IDMEC - Instituto Superior Técnico, Universidade de Lisboa, Av. Rovisco Pais, 1, 1049-001 Lisboa, Portugal \\ ${ }^{\mathrm{b}}$ School of Engineering and Technology, University of Hertfordshire, College Lane Campus, Hatfield, Herts AL10 9AB, UK \\ ' Escola Náutica Infante D. Henrique, Av. Eng. Bonneville Franco, 2770-058, Paço d'Arcos, Portugal
}

\section{A R T I C L E I N F O}

\section{Article history:}

Received 14 May 2016

Received in revised form 15 July 2016

Accepted 18 August 2016

Available online xxx

\section{Keywords:}

Fatigue

Files

NiTi wires

Life evaluation

\begin{abstract}
A B S T R A C T
Nickel-Titanium (NiTi) alloys with superelastic properties have been increasingly introduced as a substitute to more conventional alloys, such as stainless steel, in a variety of applications. In Dentistry, NiTi alloys are used in tools such as Endodontic rotary files, allowing the file to follow teeth root canals more easily than their stainless steel counterparts. Nevertheless, during surgery, the file is subjected to cyclic bending loading, since it is rotating while being deformed inside the curved canals and is prone to fracture due to fatigue, without showing any visible signals of degradation. Following a systematic approach, this work presents the results of rotary fatigue tests for several NiTi wires from different manufacturers (Memry and Euroflex). The formulation is presented, where the material strength reduction can be quantified from the determination of the strain and the number of cycles until failure. Experimental tests as well as numerical Finite Element Analysis (FEA) simulations are presented to better understand the fatigue fracture mechanisms present in NiTi alloys, showing that there is good agreement between the predicted strains (difficult to measure in such small wires) and the cycles to failure.
\end{abstract}

(C) 2016 Published by Elsevier Ltd.

\section{Introduction}

In dentistry, the root canal procedure is done using a rotary file that removes the existing nerve endings on a tooth. In the past, endodontic files used in this procedure were made from highly flexible steel alloys. However, steel alloy files, while being flexible, are still too rigid to avoid damaging the walls of the root canals. In order to minimize these adverse effects, Nickel-Titanium alloys are used in the design of endodontic rotary files instead of stainless steel alloys. NiTi alloys are superelastic metal alloys that are able to fully recover from large deformations (up to strains of 10\% [1]). These alloys, however, have a drawback when compared to steel files: their fatigue life is relatively shorter than steel and, as seen in commercial endondontic files, they break without a previous mechanical warning, increasing the risk of the file failing inside the teeth.

There are some studies to determine the fatigue life of NiTi alloys, through traditional uniaxial fatigue tests and rotary bending fatigue tests [2]. Rotary bending tests are the tests that most accurately replicate the kind of loads and deformation a file is subjected to when inside a root canal. The great majority of the existing machines in the literature only perform the fatigue test with a predetermined set of shapes. However, most of the imposed deformations are far from the complex shapes of the root canals [2].

Of special interest is the rotary fatigue machine designed by Cheung and Darvell [3]. This machine consists of three pins that can be positioned manually to deform the endondontic file. The file is then put into rotation using a contra-angle. This type of machine has an advantage of being more versatile than the more common machines

\footnotetext{
* Corresponding author.

Email address: mfreitas@dem.ist.utl.pt (M. Freitas)
}

with simulated canal carved in a stainless steel plate, where one can have only one predetermined curvature per plate [4-7].

In this work, an automated configurable rotary bending-testing machine was designed. This testing machine was designed to adapt and change the degree of bending from simple point bending to more complex multi-point bending. The machine consists in three pairs of pins positioned by servomotors, which deform the specimen into a desired complex shape. The specimen is then put into rotation until failure is detected.

The machine design also enables rotary bending tests with constant curvature (constant strain) along a segment. With a constant strain, one can compare directly the result with the more common uniaxial fatigue tests. Also, one can perform tests in different regions of the superelastic stress-strain curve, enabling an estimation of the stress and the metallic phase of the alloy during the test.

\section{Designing the testing machine}

The testing machine was designed to be versatile and to offer a wide range of possible bending configurations. Based in the machine designed by Cheung and Darvell [3], the testing machine has three pins that can be configured to make $1-4$ point bending tests. In our machine, however, the pins are positioned at configurable locations using servo-motors. Configurable positioning enables a greater precision and repeatability, while providing a simple interface (Fig. 1).

For the rotation of the specimens, a brushless DC motor with variable speed is used instead of the more common contra-angle. With a standalone DC motor, one can control the velocity of the test more precisely and automatically. The motor is able to run tests from $100 \mathrm{rpm}(1.67 \mathrm{~Hz})$ to $3000 \mathrm{rpm}(50 \mathrm{~Hz})$, which covers the range of 


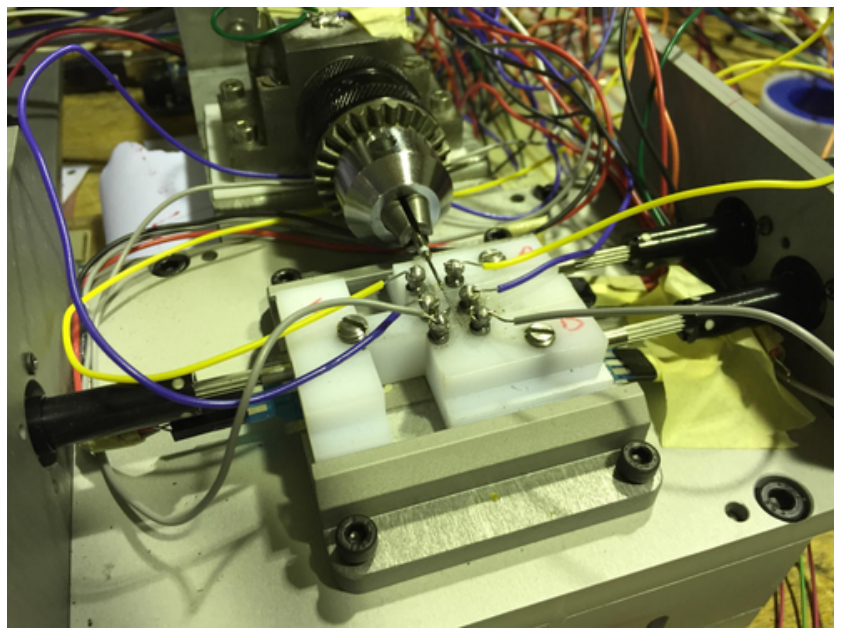

Fig. 1. Testing area with an endondontic file.

most NiTi endodontic files with a drive speed usually between 150 and $350 \mathrm{rpm}$.

To detect when the specimen fails (whether it is wire or an endondontic file), an electronic failure detection system was implemented. This system uses the natural conductivity of NiTi alloys to detect any failure by constantly monitoring the level of voltage between the specimen and each pin. When the circuit is open it means that the specimen failed and the test stops automatically.

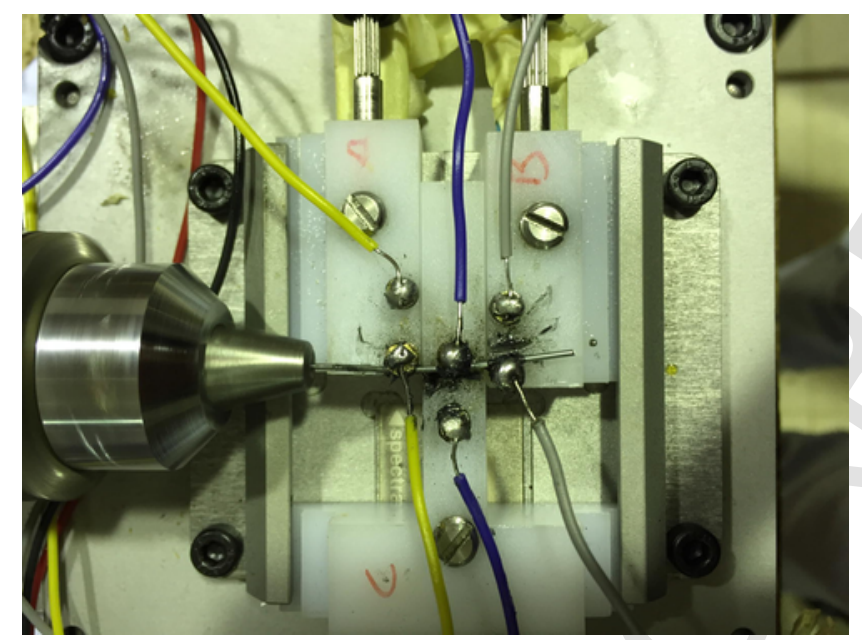

Fig. 2. Wire deformed during a test.

\section{Positioning control and constant curvature}

The most important aspect of this machine is its ability to generate bending configurations automatically. The configurations are set by positioning the pins (Fig. 1) in order to deform the specimen into a shape (Fig. 2). The pin positions are controlled using three independent PID controllers tuned for reference following (the reference here is the desired position of each pin in time).

The machine software enables two positioning modes: individual pin positioning and constant curvature radius generation.

\subsection{Constant curvature generation for wire specimens}

The main advantage of having a constant curvature in a uniform beam is that in that region the strain is also constant and proportional to the radius as in (1).

$$
\epsilon_{x}=-z \frac{1}{\rho}
$$

where $\rho$ is the radius of curvature, $\epsilon_{x}$ is the strain along the length of the beam and $z$ is the distance to the neutral surface.

Using the Euler-Bernoulli beam theory to obtain the analytical model of the wire, we obtain the deflection for the wire. For an infinitesimal element (1) becomes:

$$
\epsilon_{x}(x)=-z \frac{\mathrm{d}^{2} w}{\mathrm{~d} x^{2}}
$$

However, we want a constant strain level for an entire segment of the specimen and, consequently, the position of the pins must be determined in order to generate a constant strain section.

Using a standard square minimization algorithm to find the pin displacements needed for the desired constant strain level in the specimens mid section, while minimizing the overall deformation, we obtain the three pin positions [8]. We also have to take into account the influence of thickness of the wire and the requirement that the wire must be tangent to the pins at all times. This leads to a variable distance between the contact point at the surface and the neutral surface [8]. Adding this requirement to the optimization algorithm, we obtain deformation profiles similar to the one in Fig. 3.

For the case of Fig. 2, which corresponds to setting the desired strain to $2 \%$, the curvature can be seen in Fig. 4. Knowing that the internal radius of the pins is $1 \mathrm{~mm}$ and using (1), we obtain that the angle of the arc is $\approx 11.71^{\circ}$ and the radius of curvature is $50 \mathrm{~mm}$. These two parameters can be used to compare results with testing methods such as the Pruet et al. [9].

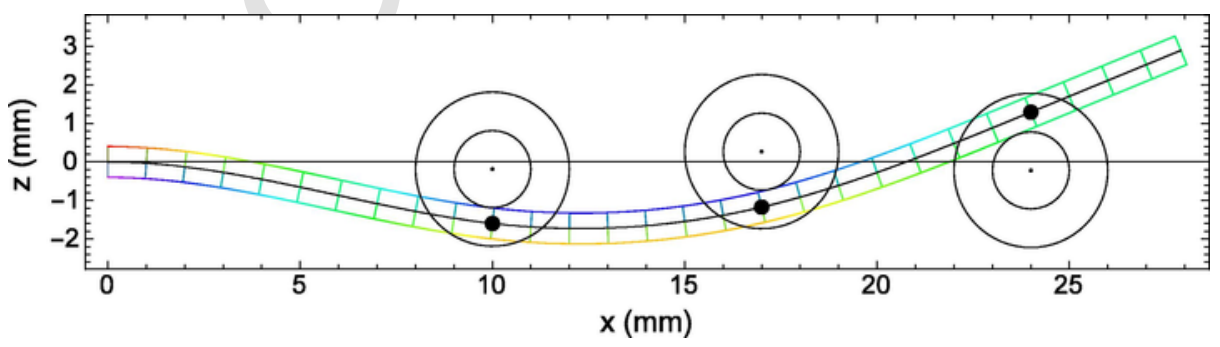

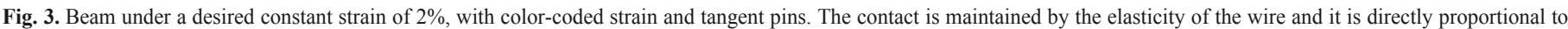
the amount of deformation. 


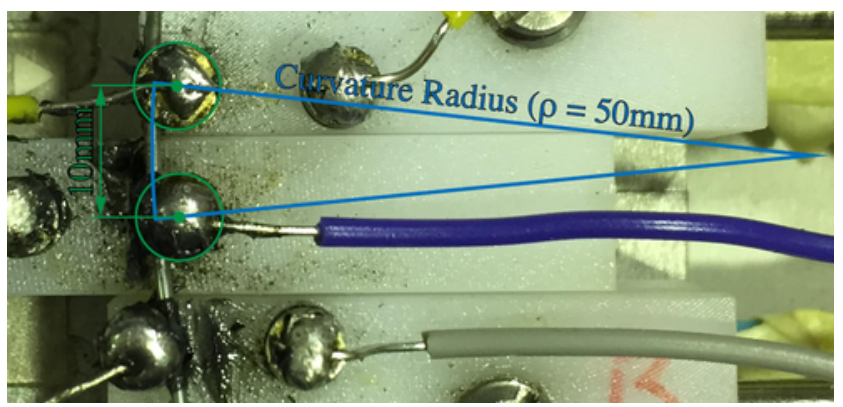

Fig. 4. Deformed wired with a desired strain of $2 \%$ superimposed with the curvature radius.

\section{Results for a NiTi wire}

The first materials to be tested in the rotary fatigue machine were a Memry ${ }^{\mathrm{TM}}$ and a Euroflex ${ }^{\mathrm{TM}}$ wires, both with a diameter of $0.8 \mathrm{~mm}$. The wires, when under an uniaxial load, have the stress-strain relation in Fig. 5.

Fig. 5 has three distinct regions: a first austenitic elastic region, a horizontal elastic region with a mixture of austenitic and martensitic phases (also known as B19' martensite phase) and a final plastic martensitic region. Another characteristic of these $\mathrm{Ni}$-Ti alloys is that they exhibit a large hysteresis when unloading occurs.

The fatigue tests were concentrated on the first two regions, imposing strain level from $0.8 \%$ to $6 \%$. The results can be seen in Fig. 6 . The specimens with strains smaller or equal to $1 \%$ (corresponding to the first zone in the stress-strain plot in Fig. 5) show a large fatigue

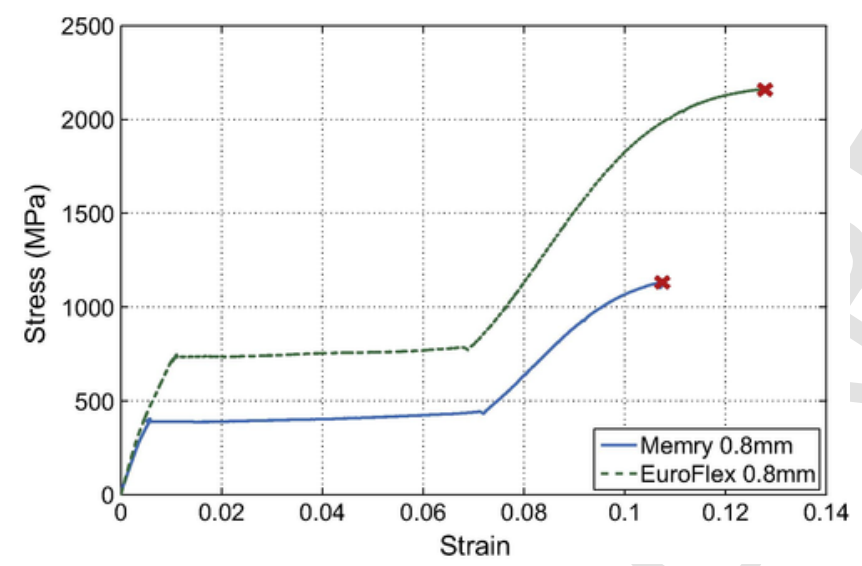

Fig. 5. Uniaxial tension test of the Memry ${ }^{\mathrm{TM}} 0.8 \mathrm{~mm}$ diameter wire.

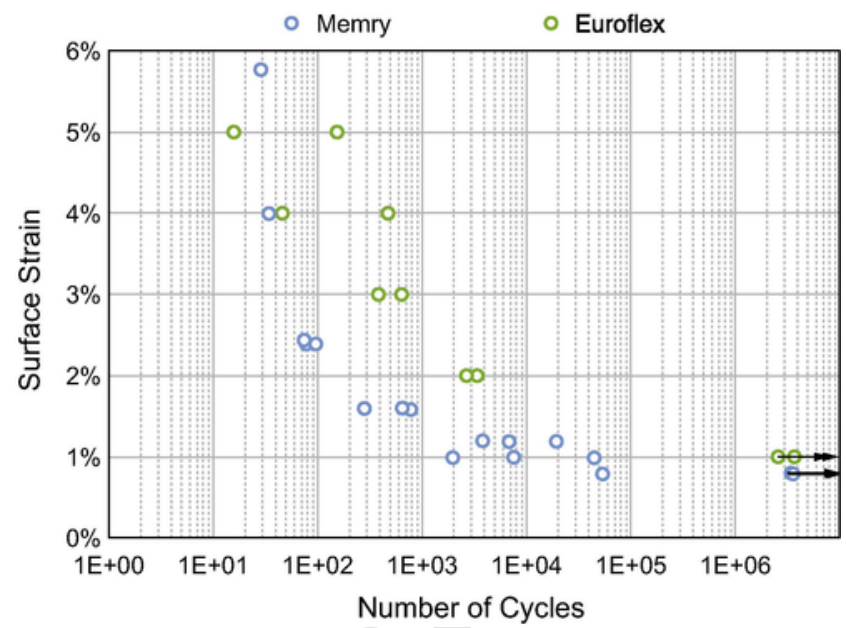

Fig. 6. Strain vs. number of cycles for a Memry ${ }^{\mathrm{TM}} 0.8 \mathrm{~mm}$ diameter wire.

life when compared with the rest of the points, with two specimens never failing. The remaining points show a decrease of the fatigue life as the imposed strain increases. The specimen with the largest strain showed a life of 34 cycles for the Memry ${ }^{\mathrm{TM}}$ wire and 16 cycle for the Euroflex ${ }^{\mathrm{TM}}$ wires.

\section{Numerical simulations}

Numerical simulations using FEA (Finite Element Analysis) can contribute to a better understanding of the failure mechanisms of the $\mathrm{Ni}$-Ti wires in the proposed testing machine. They allow predicting the stress/strain distribution through the wires cross section that could not be determined otherwise. Also, they allow determining the maximum values for the strain more accurately than the Euler-Bernoulli beam theory approximation. This is because the deformations imposed are large and the superelastic material properties are highly non-linear.

\subsection{Model}

A static structural FEA was conducted in ANSYS16.1 Workbench from the model shown in Fig. 7. The $0.8 \mathrm{~mm}$ diameter wire was modeled with 13,651 solid elements with superelastic $[8,10]$ and linear elastic isotropic material properties introduced in the project's Engineering Data cell as shown in Table 1. The wire was fixed in one end by a combination of a clamp and a fixed support covering $10 \mathrm{~mm}$ of the wire's length. The wire was deformed under bending using three $1 \mathrm{~mm}$ radius steel actuator pins that moved in the $y$ direction based on the constant curvature formulation earlier presented. The movement

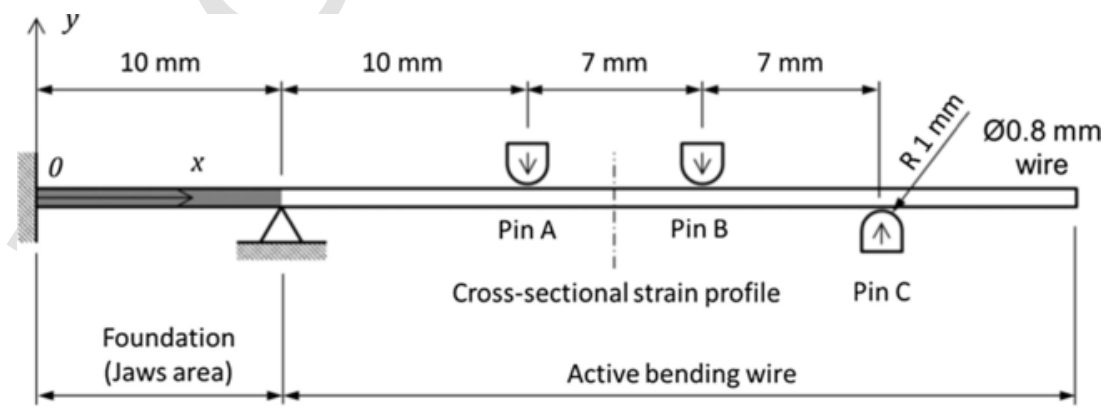

Fig. 7. Schematics of the model (geometry and boundary conditions). 
Table 1

Material properties for the Memry ${ }^{\mathrm{TM}}$ and Euroflex ${ }^{\mathrm{TM}}$ alloys introduced in ANSYS workbench projects engineering data project cell.

\begin{tabular}{lll}
\hline Material property & Memry $^{\mathrm{TM}}$ & Euroflex $^{\mathrm{TM}}$ \\
\hline $\begin{array}{l}\text { Isotropic elasticity } \\
\text { Young Modulus(GPa) }\end{array}$ & 70 & 70 \\
Poisson's Ratio & 0.33 & 0.33 \\
Superelasticity & & \\
Sigma SAS (MPa) & 368 & 736 \\
Sigma FAS (MPa) & 484 & 798 \\
Sigma SSA (MPa) & 232 & 337 \\
Sigma FSA (MPa) & 100 & 135 \\
Epsilon (mm/mm) & 0.063 & 0.059 \\
Alpha & 0 & 0 \\
\hline
\end{tabular}

of the actuators was simulated as being gradual, in an explicit iterative way. Eight different positions were considered in the analysis (Table 2 ). The relative positions of the pins in Table 2 correspond to the ones used in the actual experiments, determined from the theoretical discussion. Frictionless contact properties were added between the actuator pins and the wire.

\subsection{FEA model validation}

The FEA results were validated by a combination of different procedures, including a mesh sensitivity analysis. Initially, the model in Fig. 7 considered the whole foundation to be perfectly clamped, hence constraining rotational degrees of freedom at the fixed support location, as in a previous work [11]. However, a stress concentration was being observed at the clamp location. Furthermore, from the experimental results later presented, it was observed that the wire generally collapses either on pin A or pin B. This was inconsistent with the FEA model that was indicating the clamping location as the most stressed region. Therefore, in an attempt to better simulate the machine's jaws, the wire was fixed by a combination of a clamp and fixed support set apart $10 \mathrm{~mm}$ away from one another as shown in Fig. 7. Even if this might not still represent the fixture with the wanted accuracy, at least the locations where the maximum values for the strain (and stress) occur are now consistent with experimental data, i.e., they occur between pins $\mathrm{A}$ and $\mathrm{B}$.

The maximum values found for the strain (equivalent elastic) in the constant deformation section of the wire (between pins A and C) was determined and compared with the theoretical value from the Euler-Bernoulli beam theory. It was observed a discrepancy between these values as the strain increases, which is expected since the Euler-Bernoulli model is only valid for small displacements with constant and uniform Young Modulus. The results from this analysis are summarized in Table 3.

To better understand why the strain determined from the superelastic material model (Euroflex in Table 3) is not increasing with the bending deformation (as it would be expected), the same wire was stretched up to a theoretical strain of $5 \%(2.25 \mathrm{~mm}$ in the $x$ direction for a $45 \mathrm{~mm}$ long wire). However, and once more, the values for the

Table 2

Positions of the pins for the FEA analysis with reference to the datum in Fig. 7 ( $y$ direction).

\begin{tabular}{llll}
\hline Positions & Pin A $(\mathrm{mm})$ & Pin B $(\mathrm{mm})$ & Pin C $(\mathrm{mm})$ \\
\hline Position 1 & -0.786 & -0.535 & 0.525 \\
Position 2 & -1.572 & -1.070 & 1.050 \\
Position 3 & -2.358 & -1.606 & 1.575 \\
Position 4 & -3.144 & -2.141 & 2.10 \\
Position 5 & -3.930 & -2.676 & 2.625 \\
\hline
\end{tabular}

Table 3

Strain maximum values (in $\mathrm{mm} / \mathrm{mm}$ ) in the constant deformation section of the wire (between pins A and C) obtained from four different models, using Euroflex material properties.

\begin{tabular}{lllll}
\hline & $\begin{array}{l}\text { Euler-Bernoulli } \\
\text { beam theory } \\
\text { (clamped) }(\%)\end{array}$ & $\begin{array}{l}\text { FEA linear } \\
\text { elastic } \\
\text { (clamped) } \\
(\%)\end{array}$ & $\begin{array}{l}\text { FEA linear elastic } \\
\text { ("flexible" } \\
\text { foundation) }(\%)\end{array}$ & $\begin{array}{l}\text { FEA Euroflex } \\
\text { ("flexible" } \\
\text { foundation) }(\%)\end{array}$ \\
$\begin{array}{l}\text { position } \\
\text { Position }\end{array}$ & 1 & 1.0 & 0.88 & 0.8 \\
$\begin{array}{c}\text { Position } \\
2\end{array}$ & 2 & 2.0 & 1.6 & 1.2 \\
$\begin{array}{c}\text { Position } \\
3\end{array}$ & 3 & 3.0 & 2.4 & 1.3 \\
$\begin{array}{c}\text { Position } \\
4\end{array}$ & 4 & 3.8 & 3.1 & 1.3 \\
Position & 5 & 4.6 & 3.8 & 1.2 \\
5 & & & & \\
\hline
\end{tabular}

strain using superelastic material properties did not increase proportionally to the deformation once the material was undergoing the transformation B19' martensite. This may well be because in ANSYS and for a superelastic material the determination of the elastic strain is assumed to be linearly related to the stress [12]. Therefore, strains were determined from the deformation curvature instead.

Finally, bilinear material properties were considered in ANSYS, in a similar way as in $[13,14]$, but the results were essentially the same as from using superelastic material properties.

Therefore, from this analysis, it can be considered that the FEA results are validated. Although the flexibility of the foundation is difficult to model in the software with accuracy and might be a source of uncertainty, this will not have a detrimental effect on the conclusions that can come out from this study.

\subsection{Results}

Fig. 8 shows the deformation and the von Mises stress distribution on the beam obtained from ANSYS, considering Euroflex material properties (Table 1) and for the pins displaced at position 5 (Table 2). It can be observed that the maximum strain occurs between pins $A$ and $\mathrm{B}$ (the ones moving in the negative $y$ direction). Therefore, it is expected that the wire will collapse between these two pins.

The deflection for the beams when modeled with different material properties (Memry, Euroflex and linear elastic) was similar (Fig. 9).

Using the deformation data for the different deflections given by ANSYS for the different positions, one can determine the curvature by applying (3):

$$
\kappa=\frac{w^{\prime \prime}}{\left(1+\left(w^{\prime}\right)^{2}\right)^{\frac{3}{2}}}
$$

where $\kappa$ is the curvature and $w$ is the deflection. Note that this equation is a simplified version that assumes that there is no axial strain. ${ }^{1}$

Plotting the strain between the first and second pins (at $x=20 \mathrm{~mm}$ and $x=27 \mathrm{~mm}$, respectively) is seen in Fig. 10. Due to a more prominent effect of higher deformations and non-linearities, the

\footnotetext{
${ }^{1}$ As it will be seen below, the FEA model predicts that there will be some axial strain. However it can be safely ignored, because it is very small when compared to the bending strain.
} 


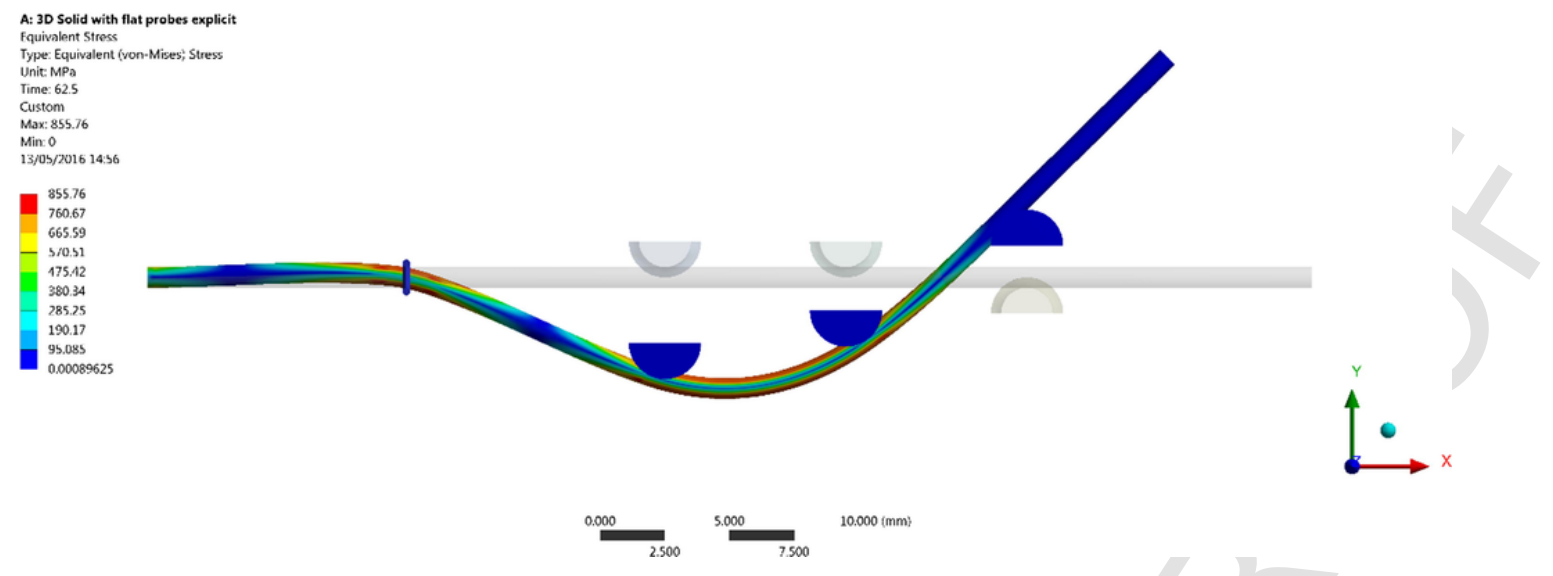

Fig. 8. Deformation and equivalent elastic strain distribution on the beam obtained from ANSYS (Euroflex, pins @ position 5).

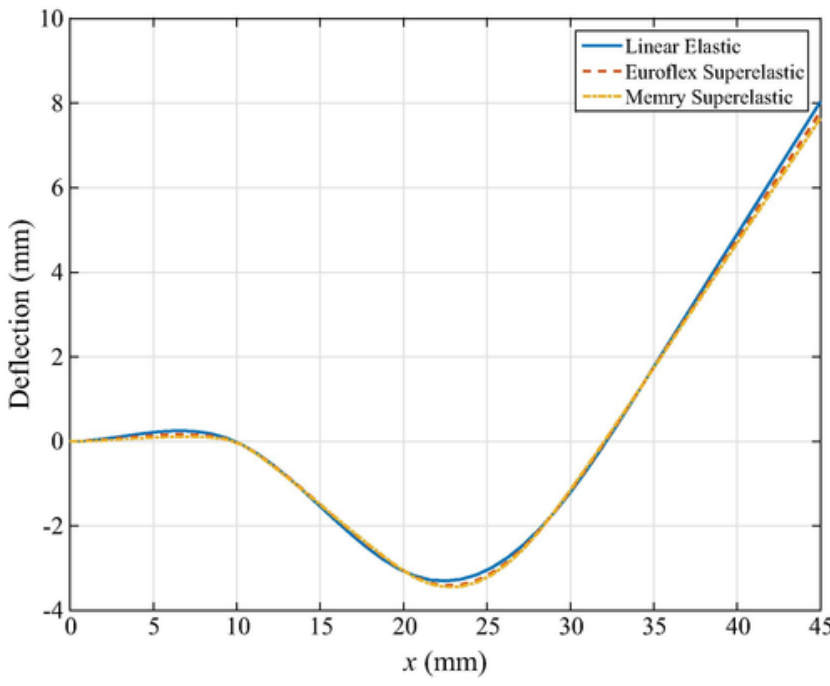

Fig. 9. Comparison between the deformation of wires made from different materials obtained from ANSYS at position 5.

strain in this zone is not constant as predicted by the Euler-Bernoulli model (although the curves become flatter as the strain level decreases).

Theoretically, the plots in Fig. 10 show that the collapse point should be at the maximum point of the strain level. This, however, is not verified experimentally since the wire always collapsed at the pins. This discrepancy is probably due to the presence of wear and tear at the pins, which increases the effects of fatigue as it generates preferential starting points for cracks.

Plotting the evolution of the maximum predicted strain by the FEA versus the one by the Euler-Bernoulli model, we obtain the relation in Fig. 11 for the linear elastic and superelastic models.

Fig. 11 shows that the relationship between the FEA strain and the Euler-Bernoulli strain is not linear, which may indicate that from a certain point onwards the wire starts stretching. However, this may also well be due to the fixture in the FEA not being a perfect clamp. Therefore, to better understand why this is happening, it is convenient to look into how the stress develops across the beams cross-section. Taking as a reference the equidistant section from pins A and B (indicated by the dash-dot line in Fig. 7, located at $x=23.5 \mathrm{~mm}$ ), the stress profiles for Euroflex, Memry and linear elastic material properties take the forms represented in Figs. 12-14, respectively.
Euroflex stress profile in Fig. 12 for pins at position 1 is "V" shaped as are the stress profiles when using the linear elastic isotropic formulation (Fig. 14). However, from position 2, the stress profile becomes "bird" shaped for both the Euroflex and Memry alloys, with a slight increase in stress near the neutral line (at the center in the graphs) and the wire cross-sectional circular boundary (at the left and right extremes in the plots). This is consistent with the assumption that the wire is slightly stretching. However, the non-linearity observed in Fig. 11 is most likely related to the fact that the boundary conditions are softer in the FEA model than in the Euler-Bernoulli one, since the FEA model allows some rotation at the fixture. The linear elastic wire, showing a larger curvature at the fixture location than the superelastic wires (Fig. 9), therefore produces less linear results in Fig. 11. Finally, comparison of the superelastic stress profiles (Figs. 12 and 13) with the linear elastic stress profile (Fig. 14), where the latter maintains a "V" shaped profile whereas the former change to "bird" shaped profiles, suggests that the Ni-Ti alloy presents two crystallographic phases at the same time:

1. The part of the wire cross-section closer to the neutral line is still Austenitic, whereas;

2. The parts of the wire cross-section further away from the neutral line are going through the Austenite to B19' Martensite crystallographic transformation intermediate step characteristic from Ni-Ti superelastic alloys [15-18].

Using the new predicted strains from the FEA model, we can update the experimental plot of the fatigue life of the Memry and Euroflex wires (Fig. 15).

Of special note is that the infinite life for the Euroflex is only obtained in the linear elastic phase.

\section{Fracture surfaces}

The fracture surfaces of the specimens tested in the rotary testing bending machine were observed on a Scanning Electronic Microscopy, JEOL existent at MicroLab from IST. For comparison purposes, the fracture surface of the specimens tested in uniaxial tension was also observed. Fig. 16 show respectively, the general view of the fracture surface of the uniaxial tension specimen Memry ${ }^{\mathrm{TM}} 0.8 \mathrm{~mm}$ and the amplification of the fracture surface where a characteristic ductile facies can be observed with dimples developed during the failure process.

The fracture surfaces observed in fatigued specimens are substantially different from the uniaxial testing ones and are characterized by 

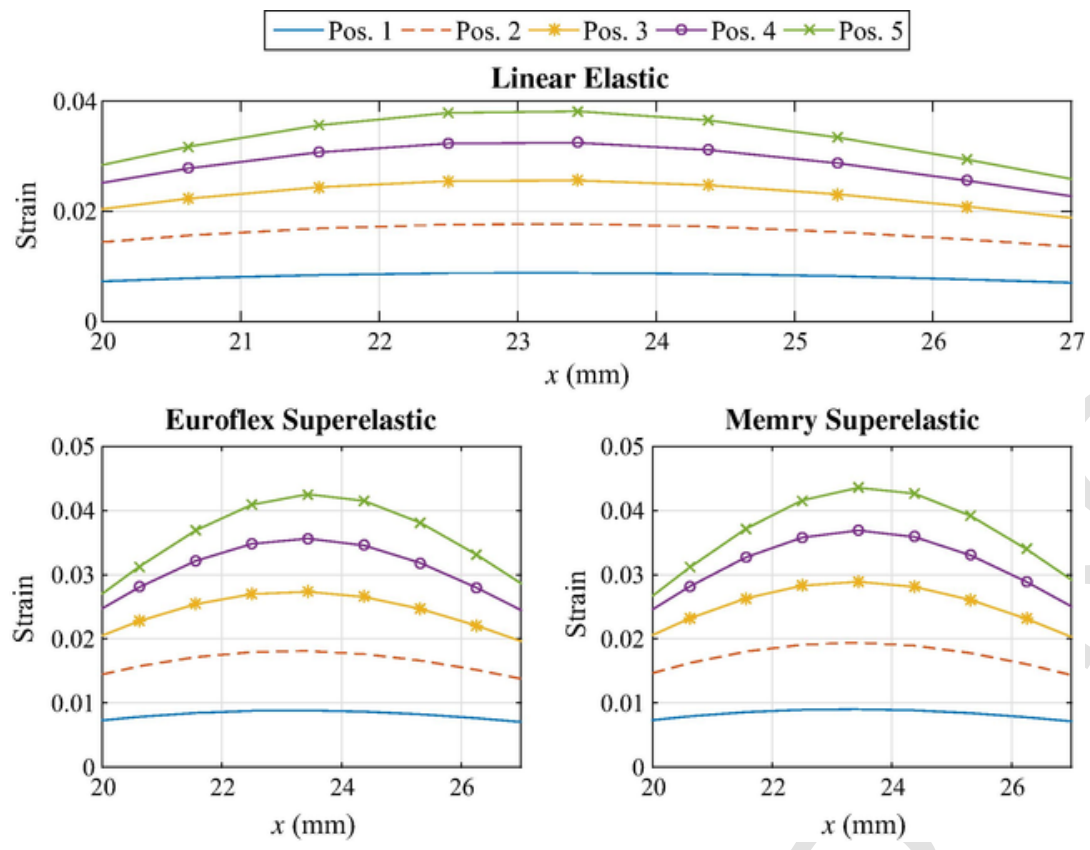

Fig. 10. Surface strain between the first and second pins for different models and different pin positions.

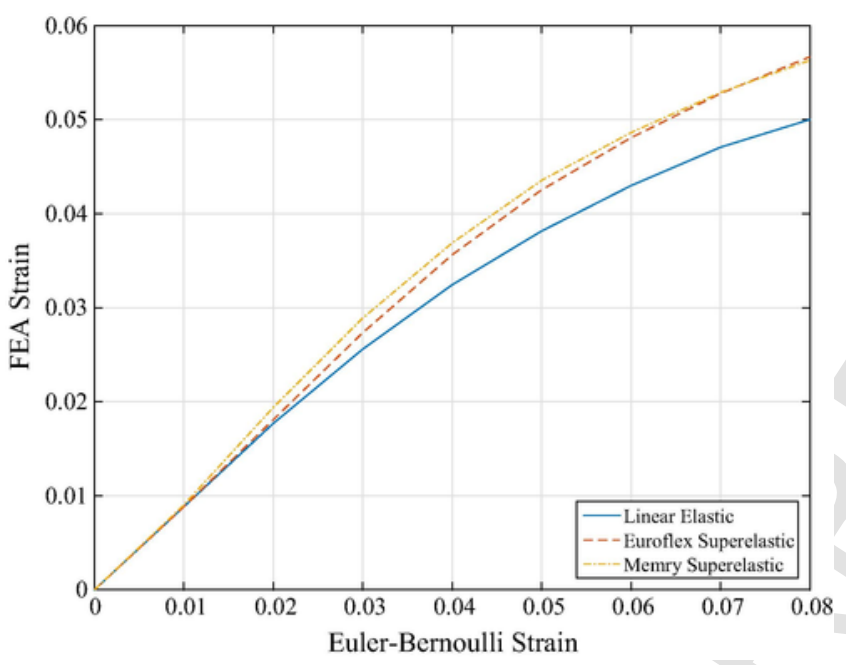

Fig. 11. Maximum FEA strain vs Euler-Bernoulli strain.

flat fractures surfaces, as usually observed in failed specimens under rotating bending conditions. For lower cycles to failure a very small fatigue zone is observed near the surface. As the number of cycles to failure increases, the fatigue zone on the specimens also increases. Fig. 17a shows the fractured surface of the specimen of Memry ${ }^{\mathrm{TM}} 0.8 \mathrm{~mm}$ failed by fatigue at 44,551 cycles. A clear focus of crack initiation can be observed and is shown in detail on Fig. 17b which is significantly different from Fig. 16b. Therefore these NiTi alloy fractured at rotating bending fatigue do not differ from fractured surfaces by fatigue in more classic isotropic metals.

\section{Conclusions and future work}

The work presented on this paper presents a testing apparatus designed to perform rotary fatigue tests on NiTi endondontic files and wires under different loading conditions. The testing machine was

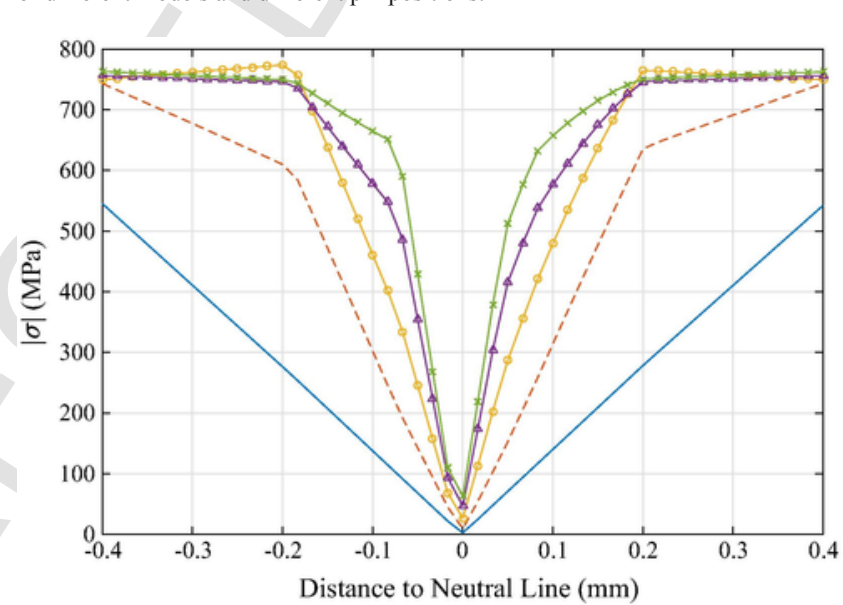

-Position 1 ---Position $2-0-$ Position $3 \rightarrow$ Position $4 \rightarrow-$ Position 5

Fig. 12. Absolute valued stress profile across the beam cross section between pins $A$ and B (Euroflex).

designed to be versatile, enabling a series of testing configurations. Computer controlled automatic positioning of a system comprised of three pins is used to bend the specimens into many user specified shapes. A combination of analytical and numerical models allows determining the strain (and stress) levels at different locations of the NiTi wires. This would otherwise be very hard to measure due to the wires very small thickness. A series of fatigue tests were done using a Memry and Euroflex $0.8 \mathrm{~mm}$ diameter wires. However, a bending configuration that has a constant maximum strain section was imposed (between pins A and B), allowing for S-N curves to be plotted. The wires showed a very long fatigue life (most of the specimens did not fail) when under strain levels in the elastic austenitic phase. When imposing strains in the B19' martensite region, the fatigue life of the wire drastically reduced, with fatigue life ranging from 20,000 (lower strain levels) to 16 cycles (higher strain levels). Finally, observation of the fracture surfaces using SEM showed that these NiTi alloys 


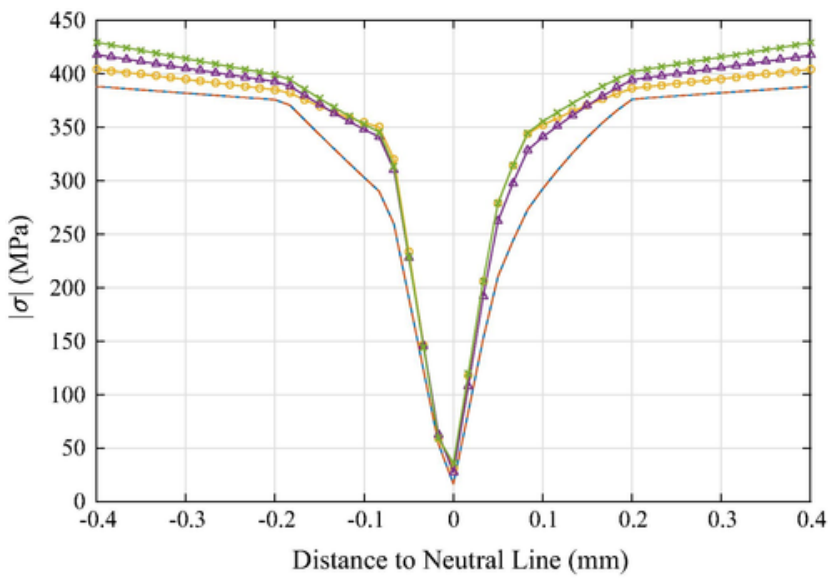

-Position 1 ---Position $2--$ Position $3 \rightarrow-$ Position $4 \rightarrow-$ Position 5

Fig. 13. Absolute valued stress profile across the beam cross section between pins A and B (Memry).

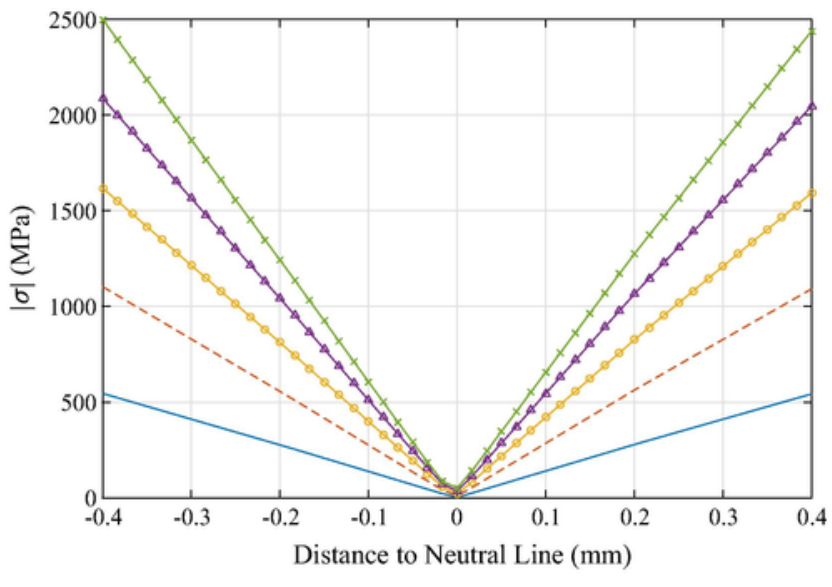

-Position $1---$ Position $2-0-$ Position $3-\triangleleft-$ Position $4 \rightarrow-$ Position 5

Fig. 14. Absolute valued stress profile across the beam cross section between pins A and B (linear elastic).
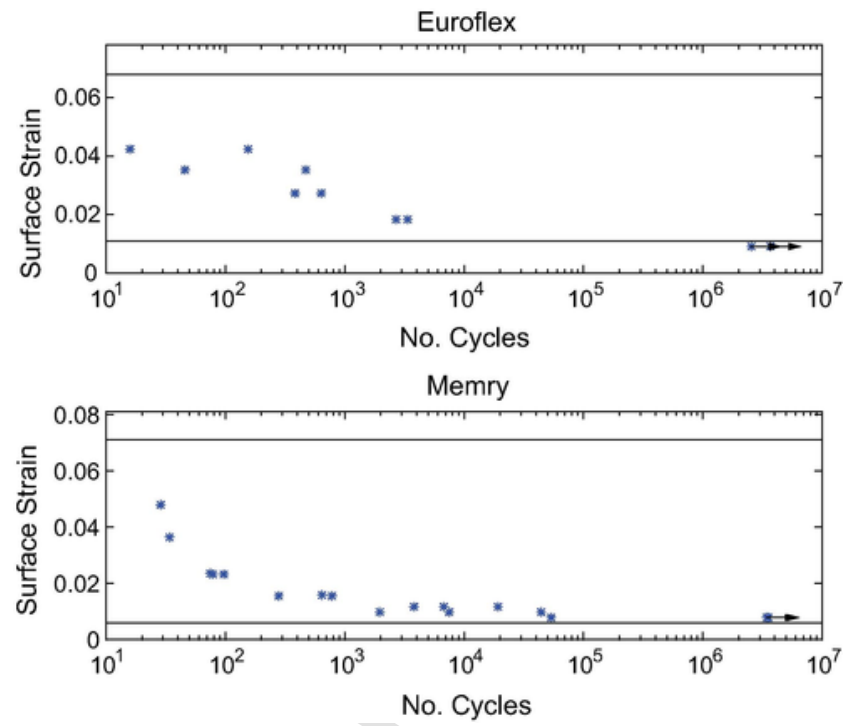

Fig. 15. Fatigue lives of the two wires with FEA corrected strain. The horizontal lines mark the beginning and end of the B19' martensite phase.

fracture mechanisms do not differ significantly from the ones observed in more traditional isotropic metals. For future work, other wires with different diameters will be tested, as well different Endondontic files.

\section{Acknowledgments}

This work was supported by national funds through FCT PortugalFundação para a Ciência e a Tecnologia as part of the project PTDC/ EME/AME/122795/2010.

Authors would like to thank the Portuguese Foundation for Science and Technology through IDMEC, under LAETA, project UID/EMS/ $50022 / 2013$.

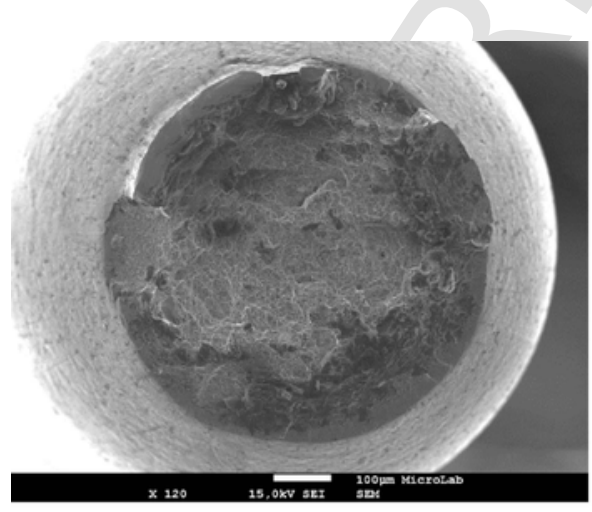

(a) General view

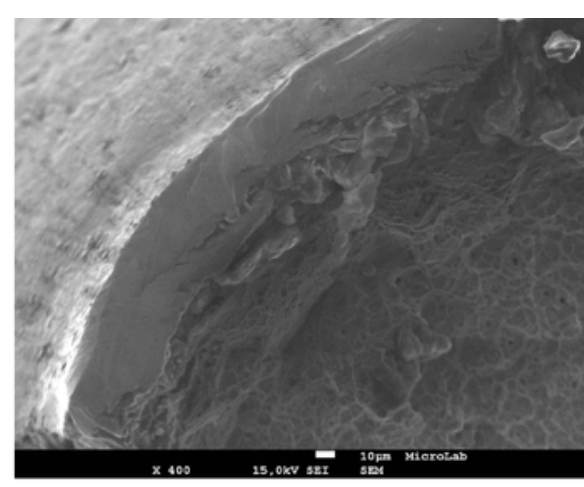

(b) Amplification of the fracture surface

Fig. 16. Uniaxial tension 


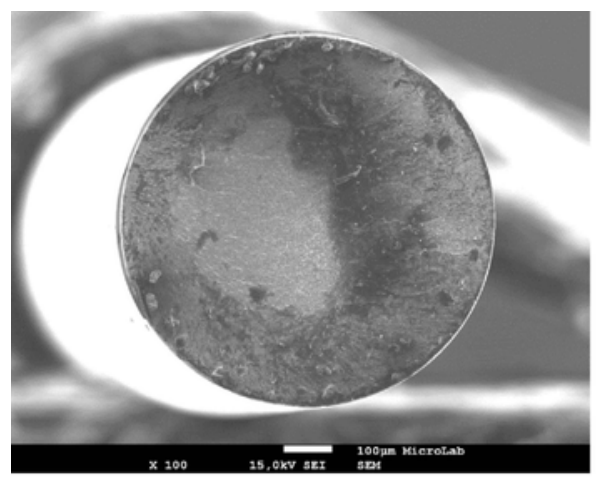

(a) Failure at 44551 cycles

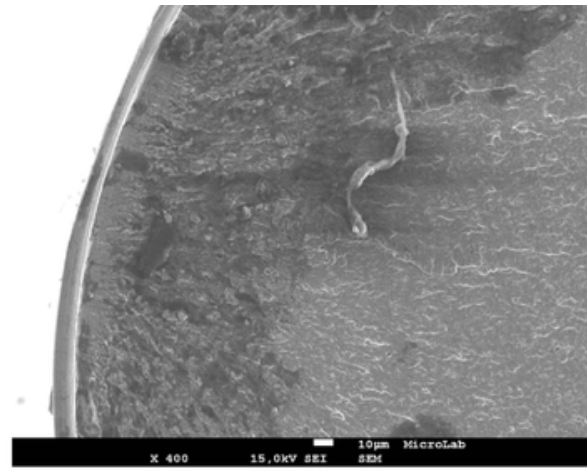

(b) Crack initiation

Fig. 17. Rotary bending fatigue.

\section{References}

[1] S.W. Robertson, A.R. Pelton, R.O. Ritchie, Mechanical fatigue and fracture of nitinol, Int. Mater. Rev. 57 (1) (2012) 1-36.

[2] G. Plotino, N.M. Grange, M. Cordaro, L. Testarelli, G. Gambarini, A review of cyclic fatigue testing of nickel-titanium rotary instruments, Cyclic Fatigue Rotary Instrum. 35 (1) (2009) 1469-1476.

[3] G.S.P. Cheung, B.W. Darvell, Fatigue testing of a niti rotary instrument. Part 1: Strain-life relationship, Int. Endodontic J. 40 (2007) 612-618, http://dx.doi.org/ 10.1111/j.1365-2591.2007.01262.x.

[4] G. Plotino, N.M. Grange, M.C. Melo, M.G. Bahia, L. Testarelli, G. Gambarini, Cyclic fatigue of niti rotary instruments in a simulated apical abrupt curvature, Int. Endodontic J. 43 (2010) 226-230, http://dx.doi.org/10.1111/j.1365-2591. 2009.01668.x.

[5] H.P. Lopes, I.M.O. Britto, C.N. elias, J.C.M. de Oliveira, M.A.S. Neves, E.J.L. Moreira, et al., Cyclic fatigue resistance of protaper universal instruments when subjected to static and dynamic tests, Oral Surg. Oral Med. Oral Pathol. Oral Radiol. Endod. 110 (2010) 401-404, http://dx.doi.org/10.1016/j.tripleo.2010.05. 013.

[6] G. Gambarini, R. Gergi, A. Naaman, N. Osta, D. Al Sudani, Cyclic fatigue analysis of twisted file rotary niti instruments used in reciprocating motion, Int. Endodontic J. 45 (2012) 802-806, http://dx.doi.org/10.1111/j.1365-2591.2012. 02036.x.

[7] G. De-Deus, E.J.L. Moreira, H.P. Lopes, C.N. elias, Extended cyclic fatigue life of F2 ProTaper instruments used in reciprocating movement, Int. Endodontic J. 43 (2010) 1063-1068, http://dx.doi.org/10.1111/j.1365-2591.2010.01756.x.

[8] A. Carvalho, M. Freitas, L. Reis, D. Montalvão, M. Fonte, Rotary fatigue testing machine to determine the fatigue life of NiTi alloy wires and endodontic files, Proc. Eng. 114 (2015) 500-505, http://dx.doi.org/10.1016/j.proeng.2015. 08.098 .

[9] J. Pruett, D. Clement, D. Carnes, Cyclic fatigue testing of nickel titanium endodontic instruments, J. Endodontics 23 (1997) 77-85.

[10] W. Yin, F. Sczerzenie, M. Long, C. Belden, R. Manjeri, R. Lafond, Structure and properties of large diameter hot rolled NiTi bars for seismis applications, in: SAES Smart Materials, New Harthford, New York, 2015.

[11] A. Carvalho, M. Freitas, D. Montalvão, M. Fonte, Rotary fatigue testing to determine the fatigue life of NiTi alloy wires: an experimental and numerical analysis, Proc. Struct. Integr. 1 (2016) 34-41.

[12] F. Auricchio, J. Lubliner, A uniaxial model for shape-memory alloys, Int. J. Solids Struct. 34 (27) (1997).

[13] D. Montalvão, F.S. Alçada, Numeric comparison of the static mechanical behavior between ProFile GT and ProFile GT Series X rotary nickel-titanium files, J. Endodontics 37 (8) (2011) 1158-1161.

[14] X. Xu, M. Eng, Y. Zheng, D. Eng, Comparative study of torsional and bending properties for six models of nickel-titanium root canal instruments with different cross-sections, J. Endodontics 32 (2008) 372-375.

[15] A. Nagasawa, A new phase transformation int the NiTi alloy, J. Phys. Soc. Jpn. 29 (5) (1970) 1386

[16] H.C. Ling, R. Kaplow, Macroscopic length changes during the B2 $\leftrightarrow$ R and $\mathrm{M} \leftrightarrow \mathrm{B} 2$ transitions in equiatomic NiTi alloys, Mater. Sci. Eng. 51 (2) (1981) 193-201.

[17] A.S. Paula, K.K. Mahesh, C.M.L. Santos, J.P.H.G. Canejo, F.M.B. Fernandes, One- and two-step phase transformation in Ti-rich NiTi shape memory alloy, Int. J. Appl. Electromagnet. Mech. 23 (1-2) (2006) 25-32.

[18] D. Montalvão, F. Alçada, F. Fernandes, S. Correia, Structural characterisation and mechanical $\mathrm{FE}$ analysis of conventional and $\mathrm{M}$-wire $\mathrm{Ni}-\mathrm{Ti}$ alloys used in endodontic rotary instruments, Sci. World J.: Mater. Sci. (2014). 